\title{
GEODESICALLY COMPLETE HYPERBOLIC STRUCTURES
}

\author{
ARA BASMAJIAN AND DRAGOMIR ŠARIĆ
}

\begin{abstract}
In the first part of this work we explore the geometry of infinite type surfaces and the relationship between its convex core and space of ends. In particular, we give a geometric proof of a Theorem due to Alvarez and Rodriquez that a geodesically complete hyperbolic surface is made up of its convex core with funnels attached along the simple closed geodesic components and half-planes attached along simple open geodesic components. We next consider gluing infinitely many pairs of pants along their cuffs to obtain an infinite hyperbolic surface. Such a surface is not always complete; for example, if the cuffs grow fast enough and the twists are small. We prove that there always exists a choice of twists in the gluings such that the surface is complete regardless of the size of the cuffs. This generalizes the examples of Matsuzaki.

In the second part we consider complete hyperbolic flute surfaces with rapidly increasing cuff lengths and prove that the corresponding quasiconformal Teichmüller space is incomplete in the length spectrum metric. Moreover, we describe the twist coordinates and convergence in terms of the twist coordinates on the closure of the quasiconformal Teichmüller space.
\end{abstract}

\section{INTRODUCTION}

A pair of pants is a metrically complete hyperbolic surface of type $(0,3)$ with boundary components being either closed geodesics, called cuffs, or punctures, and at least one boundary component being geodesic. The pair of pants is made geodesically complete by attaching appropriate size funnels to each geodesic boundary component. A natural way of creating more complicated hyperbolic surfaces is to glue pairs of pants along their cuffs via an isometry (where the cuffs that are glued necessarily have the same length). The gluings are not unique and they depend on a real parameter called the twist.

Recall that a Riemannian manifold is geodesically complete if and only if it is metrically complete. On the other hand, if the manifold is not metrically complete or has boundary it is natural to ask if it has a geodesic completion. Alvarez and Rodriguez (see [5]) showed that a hyperbolic surface constructed from gluing pairs of pants that form a pants decomposition $X^{\prime}$ has a unique metric completion to the convex core of a geodesically complete hyperbolic surface $X$ by attaching funnels and closed half-planes; conversely, any geodesically complete hyperbolic surface is obtained by attaching funnels and half-planes to the convex core of the surface. We state this as (Theorem 3.4) and supply our own proof. We sometimes say

The first author was supported in part by a PSC-CUNY Grant and a Simons foundation grant; the second author was supported by the National Science Foundation grant DMS 1102440, PSC-CUNY grant, and a Simons foundation grant. 
that $X$ is the geodesic completion of $X^{\prime}$. The existence of half-planes glued to the convex core appears in $[6$.

To understand the relationship between the convex core boundary and the space of ends we are led to the notion of a visible end. An end is visible if there is an open sets worth of tangent vectors that when exponentiated exit the end. A geodesic ray that begins in $C(X)$ and exits a visible end $e$ must intersect a boundary component of the convex core $C(X)$; such boundary components are called the components of the visible end e (see section 2.2 for the definition). For a finite type surface, as is well-known, a boundary component of the convex core is a simple closed geodesic which bounds a funnel. The funnel determines the visible end. More generally for an infinite type surface a boundary component of the convex core may be a simple geodesic isometric to the real line which bounds a half-plane (see Corollary 3.6 and table 2). The half-planes determine visible ends. That there can be several half-planes determining the same visible end is illustrated by our flute surface examples in section 4. A flute surface is a sequence of pairs of pants glued in succession along common length boundaries. The flute surface is not necessarily geodesically complete but always has a natural geodesic completion by Theorem 3.4. It is said to be a tight flute surface if in addition all the pants holes that have not been glued along are in fact cusps. The flute surface has a unique infinite type end-it is the limit of the isolated ends. See section 2 for the basics on ends and section 5 for more on flute surfaces (also cf. [5], 6], [7, 8], [12] and figure 4).

Let $\Omega$ be a hyperbolic domain in the complex plane. In the paper 5 it is shown that a topological half disc in $\Omega$ bounding a maximal interval on $\partial \Omega$ can be straightened to be a maximal hyperbolic half-plane. We study such a situation when $\Omega$ is the unit disc minus a countable set of points that accumulate on the boundary. In particular we prove for any $n \in \mathbb{N} \cup\{\infty\}$ there exists a tight flute surface whose unique infinite type end is a visible end with $n$ components. Though this can be proven using the work of [5] in section 4 we give a geometric proof using the convex core and the notion of visible ends. (see Theorem 4.5),

The half-planes in the above discussion arise for infinite type ends that are not metrically (or equivalently not geodesically) complete. Namely, if we glue infinitely many pairs of pants we obtain an infinite surface which might not be complete as a metric space. Indeed, when the lengths of the cuffs of the glued pairs of pants are going to infinity the distance between two cuffs is going to zero. If we choose the twists to be zero and if the distances between cuffs add up to a finite number then the obtained surface has an open finite length geodesic which leaves every compact set and thus the surface is not metrically complete (see [6]). Thus a natural question is whether there is a choice of twists such that the surface is complete regardless of how large the cuffs are. On his way to showing the existence of countable Teichmüller modular groups Matsuzaki (see [14]) demonstrated that such phenomena exist by demonstrating examples. We show that this is the case for all possible topologies on the infinite surfaces which arise by different patterns of gluing pairs of pants. That is, by choosing the twists judiciously, there is no need to attach half-planes to the infinite type ends since they are already geodesically complete (see Theorem 5.1). 
Theorem 1. Let $X^{\prime}$ be a (not necessarily complete) hyperbolic surface with a pants decomposition. Then there exists a choice of twists along the cuffs of the pants so that the induced hyperbolic surface $X$, after possibly adding funnels, is a geodesically complete hyperbolic surface.

Shiga [18, Allessandrini, Liu, Papadopuolos, Su, and Sun (cf. [2], [3], 4]) and others (cf. [13], [8], [17]) have studied the Teichmüller spaces (quasiconformal and length spectrum) of infinite surfaces either when there is an upper bound on the cuff lengths or when they are given by an explicit construction. These surfaces are complete either because of the upper bound on the cuff lengths or by the construction. For arbitrary surfaces built from the pairs of pants with unbounded cuffs a choice of twists might lead to an incomplete surface. A priori, one might think that being complete could impose conditions on the speed that the cuff lengths go to infinity (which may influence the Teichmüller theory). Theorem 2 says that the completeness of surfaces does not impose constraints on the speed of convergence to infinity of the cuff lengths and this opens the possibility for studying Teichmüller spaces of infinite surfaces of various topological types and geometrical shapes.

We proceed to analyze Teichmüller spaces of flute surfaces which are obtained by gluing pairs of pants in a chain with cuff lengths rapidly increasing. More precisely, we say a strictly increasing sequence is rapidly increasing if the sum of the first $n$ elements is going to infinity slower than the $(n+1)$-st element. We choose the twists using Theorem 2 such that the obtained hyperbolic surface $X_{0}$ is complete. Our main focus are the twists under the limits of the quasiconformal deformations when we fix the lengths of the cuffs.

The quasiconformal Teichmüller space $T_{q c}\left(X_{0}\right)$ consists of all quasiconformal deformations of $X_{0}$ modulo postcomposition by conformal maps and homotopy. The quasiconformal Teichmüller space $T_{q c}\left(X_{0}\right)$ has a natural metric given by the $1 / 2$ of the logarithm of the smallest quasiconformal constant in the homotopy class of a quasiconformal map. The length spectrum Teichmüller space $T_{l s}\left(X_{0}\right)$ consists of all homeomorphic transformation of $X_{0}$ such that the ratio of the lengths of the corresponding simple closed geodesics is bounded away from 0 and $\infty$. The length spectrum distance is $1 / 2$ the absolute value of the logarithm of the ratio of the lengths of the corresponding simple closed geodesics. It is a consequence of an inequality due to Wolpert (see [19]) that $T_{q c}\left(X_{0}\right) \subset T_{l s}\left(X_{0}\right)$.

The next theorem considers the closure (of the slice with fixed cuff lengths and varying twists of a geodesic pants decomposition) of the quasiconformal Teichmüller space $T_{q c}\left(X_{0}\right)$ in the length spectrum metric. We obtain that the twists can be proportional to the lengths of the closed geodesics which tend to infinity, (see Theorem 8.2).

Theorem 2. Let $X_{0}$ be a geodesically complete tight flute surface built by gluing pairs of pants with rapidly increasing cuff lengths $\left\{l_{n}\right\}$. Then the closure $\overline{T_{q c}\left(X_{0}\right)}$ of the quasiconformal Teichmüller space $T_{q c}\left(X_{0}\right)$ contains all surfaces with the Fenchel-Nielsen coordinates $\left\{\left(l_{n}, t_{n}\right)\right\}_{n}$, where $-C l_{n} \leq t_{n} \leq C l_{n}$, for $C>0$, and the lengths $\left\{l_{n}\right\}$ correspond to a marked surface in $T_{q c}\left(X_{0}\right)$.

Remark. In the above theorem and the theorems that follow the base point $X_{0}$ of $T_{q c}\left(X_{0}\right)$ corresponds to a fixed choice of twist parameters where the twists $\left\{t_{n}\right\}$ satisfy $0 \leq t_{n}<l_{n}$. 
We are able to describe the convergence in (the slice with fixed cuff lengths of ) $T_{q c}\left(X_{0}\right)$ with respect to the length spectrum metric (see Theorem 8.5).

Theorem 3. Let $X_{0}$ be a geodesically complete tight flute surface with twists $\left\{t_{n}\right\}$ and rapidly increasing cuff lengths $\left\{l_{n}\right\}$. Let $X_{k}$ be marked hyperbolic surface with cuff lengths equal to $\left\{l_{n}\right\}$ and twists $t_{X_{k}}\left(\alpha_{n}\right)=t_{n}+O\left(l_{n}\right)$. If $\lim _{k \rightarrow \infty} t_{X_{k}}\left(\alpha_{n}\right)=t_{n}$ for each $n$, then $X_{k}$ converges to $X_{0}$ in the length spectrum metric.

Using Theorem 3 we prove that the closure of $T_{q c}\left(X_{0}\right)$ is strictly larger than $T_{q c}\left(X_{0}\right)$ (see Theorem 8.6).

Theorem 4. If $X_{0}$ is a geodesically complete tight flute surface with rapidly increasing cuff lengths, then the length spectrum Teichmüller space $T_{l s}\left(X_{0}\right)$ is strictly larger than the quasiconformal Teichmüller space $T_{q c}\left(X_{0}\right)$. More precisely, $\overline{T_{q c}\left(X_{0}\right)}-T_{q c}\left(X_{0}\right)$ is non-empty.

Notation and contents. For the convenience of the reader in table 1 we gather some of the notation used in this paper. The section listed is the first place aside from section 1 that the notation appears. As a matter of convention we often use the prime notation such as $X^{\prime}$ to denote a not necessarily complete hyperbolic surface.

In section 2 we discuss the basics of the topology of surfaces including the classification of surfaces using the space of ends. Then we move to the basics of the geometry of ends (2.2). In section 3 we discuss pants decompositions and the relationship between the boundary components of the convex core and visible ends. Section 4 has examples of visible ends having more than one component. In section 5 we address the question of finding a geodesically complete structure with rapidly increasing cuffs. In Section 6 we define a flute surface whose cuff lengths rapidly increase. In section 7 we define the quasiconformal and length spectrum Teichmüller spaces. In section 8 we discuss various facts about the Teichmüller space of flute surface with rapidly increasing cuff lengths.

\section{TOPOLOGY AND GEOMETRY OF ENDS}

In this section we discuss some basics on topology and geometry, introduce the concept of a visible end and set-up notation. As references for the basics on hyperbolic geometry and discrete groups we refer to the books of Beardon [9] and Buser [10].

2.1. Topology of ends. A surface is of finite topological type if it has a finitely generated fundamental group. Otherwise we say it is of infinite topological type. The proof of the classification of infinite type surfaces can be found in a paper of Ian Richards ([15]). We refer the reader to the paper $([8])$ for a discussion on ends and notation. All surfaces in this paper are triangulable and orientable. Since we are interested in Riemann surfaces all of our surfaces satisfy these two assumptions.

Fix $X$ a topological surface with non-abelian fundamental group, and $\left\{X_{k}\right\}$ a compact exhaustion of $X$. Let $\mathcal{C}_{1} \supset \mathcal{C}_{2} \supset \cdots \supset \mathcal{C}_{k} \supset \cdots$ be a nested sequence of subsets of $X$ so that, for each $k, \mathcal{C}_{k}$ is a connected component of $X-X_{k}$. Two such sequences $\left\{\mathcal{C}_{k}\right\}$ and $\left\{\mathcal{C}_{k}^{\prime}\right\}$ are equivalent if for each subset $\mathcal{C}_{k}, \mathcal{C}_{k+n}^{\prime} \subset \mathcal{C}_{k}$ for large $n$, and vice-versa. These equivalence classes form the space of ends denoted $\mathcal{E}_{X}$. We usually use a representative sequence to 
TABLE 1. Definitions and notation

\begin{tabular}{|l|c|c|}
\hline \hline Definition & Section & Notation \\
\hline hyperbolic plane & 2 & $\mathbb{H}$ \\
\hline unit disc in complex plane & 2 & $\Delta$ \\
\hline quasiconformal Teichmüller space & 7 & $T_{q c}$ \\
\hline Teichmüller distance & 7 & $d_{T}$ \\
\hline length spectrum Teichmüller space & 7 & $T_{l s}$ \\
\hline length spectrum distance & 7 & $d_{l s}$ \\
\hline convex core & 2 & $C(X)$ \\
\hline boundary of convex core & 2 & $\partial C(X)$ \\
\hline equivalent boundary components of convex core & 2 & $b_{1} \sim b_{2}$ \\
\hline space of ends & 2 & $\mathcal{E}_{X}$ \\
\hline space of non-planar ends & 2 & $\mathcal{N}_{X}$ \\
\hline visible ends & 2 & $\mathcal{V} \mathcal{E}_{X}$ \\
\hline limit set & 2 & $\Lambda(\Gamma)$ \\
\hline set of discontinuity & 2 & $\Omega(\Gamma)$ \\
\hline$X$-length of $\alpha$ & 7 & $\ell_{X}(\alpha)$ \\
\hline flute surface & 5 & \\
\hline tight flute surface & 5 & \\
\hline rapidly increasing sequence & 6 & \\
\hline
\end{tabular}

denote the equivalence class of an end. We next describe a basis for the topology on the space of ends. Let $U$ be a connected component of $X-X_{k}$. Define,

$$
U^{*}=\left\{e \in \mathcal{E}_{X}: e=\left\{\mathcal{C}_{k}\right\} \text { and } \mathcal{C}_{k} \subset U, \text { for large } k\right\} .
$$

The set of all such $U^{*}$ form a basis for the topology of $\mathcal{E}_{X}$. The topology of $\mathcal{E}_{X}$ does not depend on the choice of compact exhaustion. The subspace $\mathcal{N}_{X} \subset \mathcal{E}_{X}$ denotes the subspace of non-planar ends; an end $e=\left\{\mathcal{C}_{k}\right\}$ is non-planar if each $\mathcal{C}_{k}$ has infinite genus. The non-planar ends form a closed subset of $\mathcal{E}_{X}$.

A homeomorphism $f: X \rightarrow Y$ between surfaces induces a homeomorphism of pairs,

$$
f_{*}:\left(\mathcal{N}_{X}, \mathcal{E}_{X}\right) \rightarrow\left(\mathcal{N}_{Y}, \mathcal{E}_{Y}\right),
$$

and hence the pair $\left(\mathcal{N}_{X}, \mathcal{E}_{X}\right)$ is a topological invariant of $X$ (called the end invariants of $X)$. If $X$ is of finite topological type then $X$ is a closed surface with $\left|\mathcal{E}_{X}\right|<\infty$ points deleted, and $\mathcal{N}_{X}=\emptyset$. More particularly, we will say that $e \in \mathcal{E}_{X}$ is a finite topological type end if $e$ is planar and isolated in $\mathcal{E}_{X}$. Otherwise, it is an infinite topological type end. Clearly, a surface is of infinite topological type if and only if there exists an end of infinite topological type. 
Theorem 2.1 (I. Richards, [15]). The orientable surfaces $X$ and $Y$ are topologically equivalent if and only if genus $(X)=\operatorname{genus}(Y)$ and $\left(\mathcal{N}_{X}, \mathcal{E}_{X}\right)$ is homeomorphic (as pairs) to $\left(\mathcal{N}_{Y}, \mathcal{E}_{Y}\right)$.

Let $e$ be an end of the topological surface $X$. We say that a sequence of compact sets $\left\{K_{i}\right\}$ exit the end $e$, if $\left\{K_{i}\right\}$ converges to $e$ in the space $X \cup \mathcal{E}_{X}$. By abuse of language, we also say that a path $\gamma:[0, \infty) \rightarrow X$ exits the end $e$ if $\gamma(t)$ converges to $e$ as $t$ goes to infinity. In the sequel, we will often be interested in surfaces with a hyperbolic structure and hence typically the $\left\{K_{i}\right\}$ will either be sequences of pairs of pants or simple closed geodesics and the paths $\gamma$ will be geodesic rays.

2.2. Geometry of ends. We denote the real part, resp. imaginary part, of a complex number $z$ by $\Re(z)$, resp. $\Im(z)$. A funnel is a hyperbolic surface with one geodesic boundary component which is isometric to $D /<z \mapsto e^{\ell} z>$, where $D=\{z \in U: \Re(z) \leq 0\}$ has the induced metric as a subspace of the upper half-plane model $U$ of the hyperbolic plane. Funnels are annuli with one geodesic boundary component whose length $\ell$ determines the funnel. A (standard) cusp is a hyperbolic surface with one horocyclic boundary component which is isometric to the quotient $\{z: \Im(z) \geq 1\} /<z \mapsto z+1>$, where again $\{z: \Im(z) \geq$ $1\}$ has the induced metric as a subspace of the upper half-plane model. It is well-known that any finite type geodesically complete hyperbolic surface has ends that are either cusps or funnels. We say that $Y \subset X$ is a geodesic subsurface of the hyperbolic surface $X$ if it is a subsurface with geodesic boundary.

A Riemannian manifold is geodesically complete if every geodesic can be extended infinitely far in both directions. Geodesic completeness is equivalent to the induced Riemannian (metric) distance being complete. A geodesically complete hyperbolic surface $X$ is the quotient of the hyperbolic plane, $\mathbb{H}$, by a torsion-free discrete non-elementary (Fuchsian) group $\Gamma$ of orientation preserving isometries. The action of $\Gamma$ on the ideal boundary of the hyperbolic plane breaks up into the limit set $\Lambda(\Gamma)$ and the (possibly empty) set of discontinuity, $\Omega(\Gamma)$. The set of discontinuity is made up of a countable union of intervals of discontinuity. It is well-known that the stabilizer in $\Gamma$ of an interval of discontinuity is either generated by a hyperbolic element or is trivial. Only the first possibility occurs if $\Gamma$ is finitely generated. That such a stabilizer can be trivial if $\Gamma$ is infinitely generated is investigated in the paper [6]. The convex core of $X, C(X)$, is the quotient of the convex hull of the limit set, $C H(\Lambda(\Gamma)) / \Gamma$. The convex core is the smallest closed convex subsurface (with boundary) which carries all the homotopy. In particular, all closed geodesics are contained in $C(X)$. Let $X=\mathbb{H} / \Gamma$ be a geodesically complete hyperbolic surface. $X$ (or $\Gamma$ ) is said to be of the first kind if $\Lambda(\Gamma)=\partial \mathbb{H}$; equivalently, $C(X)=X$. Otherwise it is of the second kind. We say that a sequence of oriented geodesics $\left\{L_{i}\right\}$ in $\mathbb{H}$ converge to the oriented geodesic $L$ if the endpoints of the $\left\{L_{i}\right\}$ converge to the endpoints of $L$. That is, the space of oriented geodesics can be identified with $\mathbb{S}^{1} \times \mathbb{S}^{1}-\{$ diagonal $\}$. Sometimes we are not interested in orientation of the geodesics and so we say $\left.\mathrm{E}_{i}\right\}$ converges to $L$ if up to changing orientations the convergence occurs. On a hyperbolic surface a sequence of geodesics $\gamma_{i}$ is said to converge to the geodesic $\gamma$ if the geodesics have lifts to the hyperbolic plane so that $\tilde{\gamma}_{i}$ converges to $\tilde{\gamma}$. 
To study the end geometry of a hyperbolic surface we introduce the notion of a visible end. An end $e$ of $X$ is said to be visible if there exists an open set $V$ in the unit tangent bundle of $X$ so that for any $v \in V$, the induced geodesic ray $g_{v}$ exits $e$. Otherwise, the end is said to be non-visible or complete. We denote the visible ends by $\mathcal{V} \mathcal{E}_{X}$. The next lemma allows us to describe a visible end in three different ways.

Lemma 2.2. Let $X$ be a geodesically complete hyperbolic surface. The following are equivalent.

(1) e is a visible end,

(2) there exists of point $x \in X$ and a cone of vectors based at $x$ so that their corresponding geodesics rays exit $e$.

(3) there exists a geodesic ray in $X$ that exits e and leaves $C(X)$ in finite time.

Proof. The equivalence of items (1) and (2) is clear. For the equivalence of (2) and (3), suppose there is a cone of vectors based at a point of $X=\mathbb{H} / \Gamma$ for which the corresponding geodesic rays exit $e$. Lifting these geodesic rays to the universal covering $\mathbb{H}$ and noting that they hit $\partial \mathbb{H}$ in an interval it is clear that this interval must be contained in one of the intervals of discontinuity of $\Gamma$. But then these geodesic rays leave $C(X)$ in finite time. The converse follows from the fact that if one such leaves $C(X)$ in finite time then there is a cone's worth that does.

If $X$ has a funnel then the end corresponding to the funnel is a visible end, and corresponds to exactly one component of the complement of $C(X)$. Recalling that an end $e$ is of finite (topological) type if it is isolated in $\mathcal{E}_{X}-\mathcal{N}_{X}$, a finite type end is not visible if it corresponds to a cusp of the surface, and visible if it corresponds to a funnel. Thus, as is well-known, we have a nice description of the end geometry of a hyperbolic surface with a finitely generated fundamental group.

Let $X$ be a geodesically complete hyperbolic surface. We next define an equivalence relation on the boundary components of $C(X)$. Namely, two boundary components $b_{1}$ and $b_{2}$ of $\partial C(X)$ are equivalent, denoted $b_{1} \sim b_{2}$, if there exist two geodesic rays based in $C(X)$ that go out the same end where one of them crosses $b_{1}$ and the other crosses $b_{2}$. This is clearly an equivalence relation and we denote the set of boundary components that are equivalent to the boundary component $b$ by $\{b\}$.

Now given $e \in \mathcal{V} \mathcal{E}_{X}$, let $\gamma$ be a geodesic ray based in $C(X)$ and exiting $e$. Then $\gamma$ must intersect a boundary component, say $b$, of $C(X)$. This defines a well-defined mapping, $B: \mathcal{V E}_{X} \rightarrow \partial C(X) / \sim$ given by $e \mapsto\{b\}$ which is easy to see is a bijection.

Thus a visible end of a geodesically complete hyperbolic surface $X$ corresponds to an equivalence class of connected components of the complement of $C(X)$. The boundary components that correspond (by the bijection) to the visible end $e$ we call the components of $e$. Of course in the case of a funnel there is exactly one component in its equivalence class. That there can be more than one component is investigated in section 4 . 


\section{PAnts DeCompositions And the Classification of ENDS}

In this section we discuss a geometric classification of ends. This is a consequence of Theorem 3.4 which is due to Alvarez and Rodriguez (see [5]). We give a different proof of this theorem which suits our point of view and leads to the geometric classification of ends. See lemma 2, section 6 of [6] for the need to use closed half-planes in order to geodesically complete the convex core. This half-plane is bounded by a simple open geodesic which is the limit of an infinite sequence of simple closed geodesics. Hence the closed half-plane is also maximal.

A topological pair of pants is a sphere with three disjoint closed discs removed. We sometimes include the three boundary circles as part of our topological pants. The context should make it clear. A geodesic pair of pants is a sphere with three disjoint closed discs removed endowed with a hyperbolic metric where the boundary curves are geodesic. We allow the possibility that the pair of pants has one or two cusps (a so called tight pair of pants). A pair of pants has a natural geodesic completion to a complete hyperbolic structure where each geodesic boundary component is completed by a funnel. By abuse of language we sometimes call the geodesically complete surface a pair of pants. More generally, any surface $X^{\prime}$ made up of a finite number of pairs of pants glued along common cuffs has a unique geodesic completion $X$ by adding funnels. Furthermore, $X^{\prime}=C(X)$. In fact, any geodesically complete hyperbolic surface with finitely generated fundamental group arises in this way. A topological pants decomposition of a surface is a locally finite decomposition by pairs of pants where the pants curve are homotopically distinct and nontrivial. A topological pants decomposition is a geodesic pants decomposition if the pairs of pants are geodesic pairs of pants. For ease of language, we will often drop the adjective geodesic before the terms "pair of pants" and "pants decomposition." The context should make it clear.

As we saw in section 2 the boundary of $C(X)$ in $X$ is the union of simple closed geodesics and simple open geodesics. Denoting the simple open ones by $\left\{L_{i}\right\}$, consider the surface with boundary $C(X)-\cup\left\{L_{i}\right\}$.

Proposition 3.1. Let $X$ be a geodesically complete hyperbolic surface. Every topological pants decomposition of $C(X)-\cup\left\{L_{i}\right\}$ can be straightened to a geodesic pants decomposition.

It is a consequence of Richards classification result ([15]) that any infinite type surface admits a topological exhaustion by finite type surfaces. Hence if $X$ is a geodesically complete hyperbolic surface this fact coupled with proposition 3.1 supplies us with a short proof of the following corollary.

Corollary 3.2. Let $X$ be a geodesically complete hyperbolic surface. Then $C(X)-\left\{L_{i}\right\}$ has a geodesic pants decomposition.

Remark 3.3. In the case that there are no open geodesics on $\partial C(X)$ corollary 3.2 is a result in [4].

The proof of proposition 3.1 follows. 
Proof. Let $X=\mathbb{H} / \Gamma$. Since any (topological) pants decomposition of a surface with boundary induces an exhaustion by finite type (topological) subsurfaces and vice-versa, it is enough to show that a topological exhaustion by finite type subsurfaces $\left\{K_{n}\right\}$ of $C(X)-\cup\left\{L_{i}\right\}$ straightens to an exhaustion by finite area geodesic subsurfaces. To see this let $Y_{n}$, for each $n$, be the subsurface $K_{n}$ with boundary curves straightened to geodesics; $\left\{Y_{n}\right\}$ is a geodesic subsurface of $C(X)-\cup\left\{L_{i}\right\} \subset X$. $Y_{n}$ is also homeomorphic to $K_{n}$, for each $n$, which already implies that the $\left\{Y_{n}\right\}$ are locally finite and hence the straightened geodesic pairs of pants are locally finite.

We are left to show that the $\left\{Y_{n}\right\}$ cover $C(X)-\cup\left\{L_{i}\right\}$. Now, by way of the isomorphism between the fundamental group of $X$ and the group $\Gamma$ there exists a torsion-free Fuchsian subgroup $\Gamma_{n}$ of $\Gamma$ so that $Y_{n}=C_{n} / \Gamma$, where $C_{n}$ is the convex hull of the limit set of $\Gamma_{n}$. Moreover the $\left\{\Gamma_{n}\right\}$ can be chosen so that $\Gamma_{n} \leq \Gamma_{n+1}$, and hence $C_{n} \subseteq C_{n+1}$. Note that since the fundamental group of $C(X)-\cup\left\{L_{i}\right\}$ is isomorphic to $\Gamma, \Gamma=<\Gamma_{n}>$ and $\Gamma$ keeps $\overline{\bigcup_{n} C_{n}}$ invariant. On the one hand, $\overline{\bigcup_{n} C_{n}}$ must be contained in the convex hull of $\Gamma$, and hence the boundary at infinity of $\overline{\bigcup_{n} C_{n}}$ is contained in the limit set of $\Gamma$. Since the limit set of $\Gamma$ is the smallest $\Gamma$-invariant non-empty closed subset of $\partial \mathbb{H}$ it must be that the boundary at infinity of $\overline{\bigcup_{n} C_{n}}$ is equal to the limit set. Hence $\overline{\bigcup_{n} C_{n}}$ is the convex hull of the limit set, and therefore $\overline{\bigcup_{n} C_{n} / \Gamma}=C(X)$. Thus the geodesic subsurfaces $\left\{Y_{n}\right\}$ exhaust $C(X)-\cup\left\{L_{i}\right\}$.

The ends of a hyperbolic surface constructed from finitely many pairs of pants (that is, a finite type hyperbolic surface) are well-known to be geometrically either cusps or funnels. For a hyperbolic surface constructed from an infinite number of pairs of pants we have,

Theorem 3.4. Let $X^{\prime}$ be a (not necessarily complete) hyperbolic surface constructed from gluing pairs of pants that form a pants decomposition of $X^{\prime}$. Then $X^{\prime}$ has a unique metric completion to the convex core of a geodesically complete hyperbolic surface $X$ so that $X^{\prime} \subset$ $C(X) \subset X$. Moreover, the geodesic completion of $X^{\prime}$ is attained by adding funnels and closed hyperbolic half-planes. Conversely, any geodesically complete hyperbolic surface is the geodesic completion of a (not necessarily complete) hyperbolic surface $X^{\prime}$ constructed from gluing pairs of pants that form a pants decomposition of $X^{\prime}$.

Proof. The pants decomposition induces an exhaustion by finite type geodesic subsurfaces $\left\{Y_{n}\right\}$. Observe that each such $Y_{n}$ being of finite type has a completion by adding funnels to boundary geodesics. Hence there exists a torsion-free Fuchsian group $\Gamma_{n}$ so that $\mathbb{H} / \Gamma_{n}$ is a complete hyperbolic surface with convex core $Y_{n}$. Denote the convex hull of the limit set of $\Gamma_{n}$ by $C_{n}$. Since $\Gamma_{n} \leq \Gamma_{n+1}$, we have $C_{n} \subseteq C_{n+1}$. Next set $C:=\bigcup_{n} C_{n}$, $\Gamma:=\lim _{n \rightarrow \infty} \Gamma_{n}=<\Gamma_{n}>$, and note that $\Gamma$ is a torsion-free Fuchsian group (see [6] for an infinite version of the combination theorem). Since the $\left\{C_{n}\right\}$ are an increasing nested sequence of domains with geodesic boundary, it must be that as $n \rightarrow \infty$ either the geodesics on the boundary $\partial C_{n}$ go to infinity or converge to a geodesic. In the later case we include the possibility that a component of $\partial C_{n}$ is a component of $\partial C_{n+k}$, for all $k \geq 0$. Thus $C$ is a convex subspace of $\mathbb{H}$ where, 
(1) the boundary of $C$ is made up of complete geodesics; that is, $C=\mathbb{H}-\bigcup D_{i}$, where the $D_{i}$ are open half-planes.

(2) the interior of $C$ is the universal cover of $X^{\prime}-\partial X^{\prime}$.

(3) a boundary component of $C$ in $\mathbb{H}$ has stabilizer in $\Gamma$ that is either generated by a hyperbolic element or is trivial. In the later case, the boundary component (geodesic) is the limit of axes of simple hyperbolic elements in $\Gamma$.

(4) $\Gamma$ keeps invariant $\bar{C}$, the closure of $C$ in $\mathbb{H}$.

(5) $\Gamma$ does not keep invariant any set smaller than $\bar{C}$.

(6) $C H(\Lambda(\Gamma))=\bar{C}$, and hence $\bar{C} / \Gamma$ is the convex core of $\mathbb{H} / \Gamma$, and the metric completion of $X^{\prime}$.

We can conclude that $\mathbb{H} / \Gamma$ is the geodesic completion of $X^{\prime}$ obtained by attaching closed half-planes and funnels. The closed half-planes coming from the half-planes $\left\{D_{i}\right\}$ in item (1).

For the converse, suppose $X$ is a geodesically complete hyperbolic surface and set $X=$ $\mathbb{H} / \Gamma$, where $\Gamma$ is a torsion-free discrete group. As a consequence of the fact that the boundary of $C H(\Gamma)$ in $\mathbb{H}$ is comprised of geodesics that are either axes of hyperbolic elements or have trivial stabilizer, we can conclude that the boundary of $C(X)$ in $X$ is made-up of closed geodesics and (infinite) open geodesics. we denote the infinite open geodesics by $\left\{L_{i}\right\}$. Then consider $X^{\prime}=C(X)-\cup\left\{L_{i}\right\}$ and note that we are retaining the closed geodesics, if any, on the boundary of $C(X)$. This surface with possible boundary, where the boundary components are simple closed geodesics, admits a topological pants decomposition, and by proposition 3.1 we straighten this pants decomposition to a geodesic pants decomposition.

For $\Gamma$ a non-elementary (that is, not virtually abelian) Fuchsian group, the stabilizer of an interval of discontinuity for $\Gamma$ is non-trivial if and only if the interval is bounded by the axis of a hyperbolic element in $\Gamma$. For trivial stabilizer we have the following characterization,

Corollary 3.5. Let $\Gamma$ be a non-elementary torsion-free Fuchsian group and $I$ an interval of discontinuity for $\Gamma$. Then $I$ has trivial stabilizer in $\Gamma$ if and only if there exists a sequence of simple hyperbolic elements in $\Gamma$ whose axes converge to the geodesic bounding $I$.

Proof. Denote by $L$, the geodesic bounding $I$. If $I$ has trivial stabilizer then Theorem 3.4 implies that there must exist a sequence of simple hyperbolic elements whose axes converge to $L$. Conversely, suppose simple hyperbolic elements have axes $\left\{L_{n}\right\}$ converging to $L$. If $L$ were the axis of a hyperbolic element $\gamma \in \Gamma$ then there would be $\gamma$-translates of $L_{n}$ that transversely intersect $\left\{L_{n}\right\}$ for large $n$. This violates the assumption that the $\left\{L_{n}\right\}$ are axes of simple hyperbolic elements. Thus the stabilizer of $L$, and hence $I$, is trivial.

Let $X^{\prime}$ be a (not necessarily complete) hyperbolic surface with a pants decomposition and note that $X^{\prime} \subset C(X) \subset X$. We have,

Corollary 3.6. (Visible ends and boundary components) Let $X$ be a geodesically complete hyperbolic surface. Then a boundary component of $C(X)$ is either 
(1) a simple closed geodesic that bounds a funnel in $X$ and corresponds to an isolated end that is visible or

(2) a simple geodesic isometric to the real line that bounds a half-plane in $X$ and corresponds to a component of a visible end of infinite type.

With the aid of this corollary, the following proposition characterizes the geometry of infinite type ends.

Proposition 3.7. Let $X^{\prime}$ be a (not necessarily complete) hyperbolic surface with a pants decomposition and $X$ its geodesic completion.

(1) The closure of $X^{\prime}$ in $X$ is $C(X)$, and hence $X$ is of the first kind if and only if $X=X^{\prime}$.

(2) Suppose $e$ is an infinite type end of $X$. Then e is not visible if and only if for any geodesic ray $\gamma$ that exits e the sequence of pants in the decomposition of $X^{\prime}$ that $\gamma$ intersects also exit $e$.

(3) $X$ is of the first kind if and only if each end of $X$ is not visible.

Proof. Item (1) follows from Theorem 3.4 .

To prove item (2), suppose $e$ is an infinite type end for $X$ that is not visible. Hence by Lemma 2.2 any geodesic ray $\gamma$ that exits $e$ and starts in $C(X)$ must stay in $C(X)$. Since the closure in $X$ of $X^{\prime}$ is $C(X)$ the geodesic ray $\gamma$ must pass through infinitely many pair of pants in the decomposition of $X^{\prime}$ that also exit $e$. On the other hand, if $e$ is a visible end then, again by Lemma 2.2, there exists a geodesic ray that exits $e$ and leaves the convex core $C(X)$ in finite time. Since this end is of infinite type it must be that this geodesic ray intersects $\partial C(X)$ in a simple open geodesic. Then by Theorem 3.4 this ray enters into a half plane embedded in $X$ and hence the infinitely many pairs of pants from $X^{\prime}$ that the ray intersects do not exit the end $e$. This proves item (2).

To prove item (3), suppose $X=\mathbb{H} / \Gamma$ is of the second kind and let $I$ be an interval of discontinuity for $\gamma$. Let $\beta \subset \mathbb{H}$ be a geodesic ray that limits to a point in $I$. Project to $X$ the half-plane determined by this interval and the geodesic ray $\gamma$, and note that the projected ray determines an end $e$ for which it exits. If the stabilizer of $I$ is infinite cyclic then the end $e$ corresponds to a funnel and hence is visual. If the stabilizer of $I$ is trivial then Theorem 3.4 implies again that the end is visible. To prove the other direction, suppose $X$ has a visible end. Then there exists a geodesic ray that leaves $C(X)$ in finite time. Hence by Corollary 3.6 this can only mean that the geodesic ray intersects the boundary of $C(X)$ and enters into a half-plane which it can not escape. Thus $X \neq C(X)$ and we conclude that $X$ is of the second kind.

We have shown that a visible infinite type end (of a geodesically complete hyperbolic surface) has an equivalance class of components of the convex core boundary being simple open geodesics with attached half-planes. Table 2 summarizes the relationship between the topology and geometry of an end. 
TABle 2. Ends: Geometry and Topology.

\begin{tabular}{|c|c|c|}
\hline \hline Top. vs. Geom. end. & Not visible (complete) & Visible (boundary components) \\
\hline finite type & cusp & simple closed geodesic \\
\hline infinite type & rays that exit end stay in $C(X)$ & equiv. class of simple open geodesics \\
\hline
\end{tabular}

Even though a surface can have an uncountable number of ends, the hyperbolic metric places restrictions on the geometry of the ends. Namely, the fact that a Fuchsian group has only a countable number of intervals of discontinuity implies that a complete hyperbolic surface has at most a countable set of ends that are visible. In fact, in section 4 we supply examples to show that a visible end can correspond to $n$ equivalent boundary components of $C(X)$ for any $n=1,2,3, \ldots$ including $n$ being the cardinality of the integers.

\section{EXamples: Visible ends With EQUiVAlent COMPONENTS.}

Recall that the components of a visible end are the boundary components of the convex core that correspond to the end. That is, these components bound half-planes that correspond to the end. In this section we construct examples to show that a visible end can have countable or any finite number of components. These examples first appeared in the paper [5]. Our examples are elementary; namely for each $n \in \mathbb{N} \cup\{\infty\}$ we construct a flute surface with the unique infinite type end being a visible end with $n$ components.

Let $A$ be a countable set of points in the unit disc $\Delta$ that accumulate to the set $K$ on the boundary $\partial \Delta$; the set $K$ is closed in $\partial \Delta$. We are interested in the plane domain $X=\Delta-A$ with its unique complete hyperbolic structure compatible with the complex structure. $X$ is a tight flute surface and we denote its unique infinite type end by $e$. We assume that $\partial \Delta-K \neq \emptyset$ and hence $\partial \Delta-K$ is the union of at most countably many open intervals $\left\{J_{i}\right\}$ on the boundary of the unit disc. Set $\widehat{X}=X \cup\left\{J_{i}\right\}$ and suppose $\Gamma$ is the torsion-free Fuchsian group so that $\mathbb{H} / \Gamma$ is conformally equivalent to $X$. We denote the covering map by $f: \mathbb{H} \rightarrow \mathbb{H} / \Gamma$.

Remark 4.1. Since the plane domain $X$ does not contain a simple homotopy class which bounds an annulus (in fact, only punctures and discs), we can conclude that the stabilizer of any interval of discontinuity is trivial. Or equivalently, $\Gamma$ moves any half-plane bounding an interval of discontinuity disjointly away from itself.

Lemma 4.2. If $\left\{z_{n}\right\}$ is a sequence in $\mathbb{H}$ that accumulates to an interval of discontinuity, then $\left\{f\left(z_{n}\right)\right\}$ must accumulate to $\partial \Delta$.

Proof. We first note that since the $\left\{z_{n}\right\}$ eventually enter into the complement of the convex hull of $\Gamma$ and $f$ restricted to any component of the complement is injective (remark 4.1), it must be that $\left\{f\left(z_{n}\right)\right\}$ must leave every compact subset of $X$. Then the only way $\left\{f\left(z_{n}\right)\right\}$ does not accumulate to $\partial \Delta$ is if there is a subsequence of $\left\{f\left(z_{n}\right)\right\}$ which converge to one of the points of $A$. But since the points of $A$ are punctures, there would have to be a 
subsequence of the $\left\{z_{n}\right\}$ that limit to a parabolic fixed point. Since a parabolic fixed point cannot be contained in an interval of discontinuity we have a contradiction.

Proposition 4.3. The covering map $f: \mathbb{H} \rightarrow X$ analytically extends to the set of discontinuity of $\Gamma$ so that the extension $F$ maps an interval of discontinuity to one of the $\left\{J_{i}\right\}$. Moreover, $F: \mathbb{H} \cup \Omega(\Gamma) \rightarrow \widehat{X}$ satisfies,

(1) $F$ restricted to any interval of discontinuity is injective,

(2) F establishes a one to one correspondence between $\Gamma$-equivalence classes of intervals of discontinuity and the intervals $\left\{J_{i}\right\}$.

Proof. Using Lemma 4.2, we can apply the reflection principle (see [1]) to conclude that $f: \mathbb{H} \rightarrow X$ has an analytic extension which maps an interval of discontinuity to one of the intervals $\left\{J_{i}\right\}$. We denote the extension by $F: \mathbb{H} \cup \Omega(\Gamma) \rightarrow \widehat{X}$. Furthermore, since $f$ restricted to any half-plane $Y$ that bounds an interval of discontinuity $I$ is injective (remark 4.1) and since $F$ is an analytic extension, it must be that $F$ is injective on $I$. This verifies item (1).

So far we have shown that $F$ injectively maps each interval of discontinuity into one of the $\left\{J_{i}\right\}$. To verify item (2), we need to show that this map is onto one of the $\left\{J_{i}\right\}$, and that each $\left\{J_{i}\right\}$ occurs as the image of an interval of discontinuity. To this end, suppose $J$ is one of the open intervals in $\left\{J_{i}\right\}$ and let $\beta$ be a simple differentiable curve in $X$ with endpoints being the endpoints of $J$. Denoting $J$ with its endpoints as $\bar{J}$ we have that $\bar{J} \cup \beta$ is the boundary of a simply connected region $X_{J} \subset X$ (cf. Figure 1 ). The set $f^{-1}\left(X_{J}\right)$ has countably many simply connected components and the restriction of $f$ to each component is a one-to-one conformal map by the simple connectedness. Fix one such component $Y$ and define $\phi: X_{J} \rightarrow Y$ by $\phi=\left(\left.f\right|_{X_{J}}\right)^{-1}$. Now let $\left\{z_{n}\right\} \in X_{J}$ such that $\left\{z_{n}\right\}$ accumulate to $J$ as $n \rightarrow \infty$. Then, since $f \circ \phi=\left.\mathrm{id}\right|_{X_{J}}$, it must be that $\left\{\phi\left(z_{n}\right)\right\} \in Y$ accumulate on $\partial \mathbb{H}$. The reflection principle applies to $\phi$ and hence there exists an analytic extension $\Phi: X_{J} \cup J \rightarrow \mathbb{H} \cup \partial \mathbb{H}$ of $\phi$. Since $\Phi$ is an analytic extension, $J$ is mapped onto the interior of an $\operatorname{arc} I^{\prime}$ of $\partial \mathbb{H}$. Furthermore since $\phi$ is injective it must be that $\Phi$ is injective on $J$, and hence $I^{\prime}$ is contained in an interval of discontinuity which we call $I$. Since by the identity theorem, $\Phi^{-1}=F$, for all $z \in Y \cup I^{\prime}$, we may conclude that $I^{\prime}=I$ and thus $F$ maps $I$ onto $J$. Since the argument above is natural with respect to the action of $\Gamma$ we have shown that each $\left\{J_{i}\right\}$ arises as the image of a $\Gamma$-equivalence class of intervals of discontinuity.

We may conclude that there is a one to one correspondence between $\Gamma$-equivalence classes of intervals of discontinuity and the intervals $\left\{J_{i}\right\}$.

Remark 4.4. The proof of theorem 4.3 shows that the curves $\beta$ in $X$ which bound the intervals $\left\{J_{i}\right\}$ can be chosen to be the boundary components of the convex core. See figure 1 for an illustration.

Note that the plane domain $X$ has exactly one infinite type end and in fact is the geodesic completion of a tight flute surface (see section 5 for the definition. By Proposition 4.3 the infinite type end is visible and has exactly $n$ components since the components correspond exactly to the intervals $\left\{J_{i}\right\}$. We have established, 


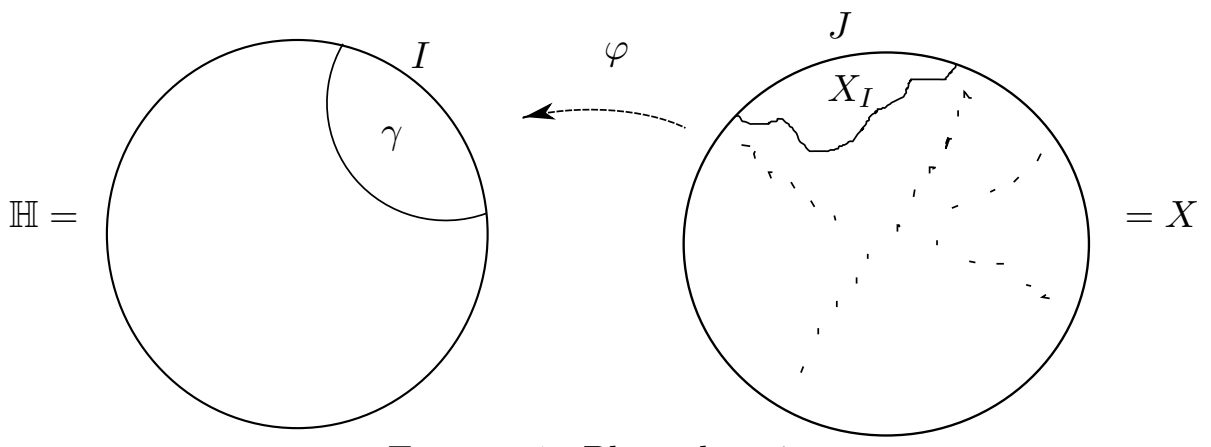

Figure 1. Plane domain.

Theorem 4.5. For any $n \in \mathbb{N} \cup\{\infty\}$ there exists a tight flute surface whose unique infinite type end is a visible end with $n$ components.

\section{Geodesically complete hyperbolic structures}

The purpose of this section is to prove the following theorem and investigate some of its consequences.

Theorem 5.1. Let $X^{\prime}$ be a (not necessarily complete) hyperbolic surface with a pants decomposition. Then there exists a choice of twists along the cuffs of the pants so that the induced hyperbolic surface $X$, after possibly adding funnels, is a geodesically complete hyperbolic surface.

Thus the induced hyperbolic surface $X$ has the property that all of its infinite ends are not visible.

We will need two lemmas for the proof of Theorem 5.1. Let $Y^{\prime}$ be a finite area hyperbolic surface with non-empty geodesic boundary, and fix a boundary component $\alpha$. Denote the geodesic completion of $Y^{\prime}$ by $Y$. We put an orientation on $\alpha$ so that the interior of $Y^{\prime}$ lies to the left of $\alpha$. We are interested in unit vectors based in $\alpha$ and directed to the interior of $Y^{\prime}$. Such a vector $v$ makes an (oriented) angle $\theta$ with $\alpha$, where $0<\theta<\pi$, and determines a geodesic ray, $g_{v}: \mathbb{R}_{\geq 0} \rightarrow Y$, we call a $\theta$-ray. A $\pi / 2$-ray is also called an orthoray. Denote the vector field of such vectors (based in $\alpha$ ) by $V_{\theta}$. Since the vectors in $V_{\theta}$ may be identified with points in $\alpha$ (take base points of vectors), $V_{\theta}$ inherits a natural measure (called the boundary measure) and topology. This topology is the same as the topology of $V_{\theta}$ as a subspace of the unit tangent bundle of $Y$. The main lemma for which the construction below hinges is the following,

Lemma 5.2. Fix $\theta, 0<\theta<\pi$. Then $V_{\theta}=A_{\theta} \dot{\cup} O_{\theta}$ where

(1) $A_{\theta}:=\left\{v \in V_{\theta}: g_{v}(t) \subset Y^{\prime}\right.$, for all $\left.t \geqslant 0\right\}$ is a Cantor set of boundary measure zero,

(2) $O_{\theta}:=\left\{v \in V_{\theta}: g_{v}(t) \cap \partial Y^{\prime} \neq \emptyset\right.$, for some $\left.t>0\right\}$ is a countable union of disjoint open intervals in $\alpha$.

Proof. $Y^{\prime}$ is the convex core of the complete hyperbolic surface, $Y=\mathbb{H} / \Gamma$, where $\Gamma$ is a torsion-free finitely generated Fuchsian group of the second kind. Consider a connected 
oriented lift of $\alpha$ to the upper half-plane and unit tangent vectors (emanating to the left) that form an angle $\theta$ with this lift. The geodesic ray determined by such a vector hits $\partial \mathbb{H}$ in exactly one point. Conversely any point on the left side of the lift of $\alpha$ in $\partial \mathbb{H}$ is the endpoint of such a geodesic ray. Hence there exists a projection map $P_{\theta}$ from $\partial \mathbb{H}$ to the lift. When $\theta=\frac{\pi}{2}$ this map is the usual orthogonal projection. The action of $\Gamma$ on $\partial \mathbb{H}$ breaks up into the (non-empty) set of discontinuity and the limit set. The set of discontinuity is made of intervals that are bounded by geodesics which are lifts of components of $\partial Y$. We next note that the geodesic ray determined by a $\theta$-vector $v \in V_{\theta}$ lifts to a geodesic ray that hits $\partial \mathbb{H}$ at a point of discontinuity if and only if the vector is in $O_{\theta}$. Otherwise the geodesic ray hits the limit set of $\Gamma$ and hence is in $A_{\theta}$. Since the limit set of a finitely generated Fuchsian group of the second kind is known to be a measure zero Cantor set we have verified items (1) and (2).

Lemma 5.3. Let $X^{\prime}$ be a (not necessarily complete) hyperbolic surface with a pants decomposition $\mathcal{P}$, and let $X$ be its geodesic completion. Fix $\gamma$ a simple closed geodesic on the hyperbolic surface $X^{\prime}$ and let e be an infinite type end of $X$. The following are equivalent,

(1) $e$ is a visible end,

(2) there exists an orthoray based in $\gamma$ that exits the end e and eventually leaves $C(X)$,

(3) there exists an interval I in $\gamma$ for which each orthoray based in I exits the end $e$ and eventually leaves $C(X)$.

Proof. We prove $1 \Longrightarrow 2 \Longrightarrow 3 \Longrightarrow 1$. If $e$ is a visible end for $X$ then by definition there exists a geodesic ray that goes out $e$ and eventually leaves the convex core $C(X)$. Hence the geodesic ray must intersect a component of the boundary of $C(X)$, denoted $\beta$, and then enter into a half-plane. By Corollary 3.6, $\beta$ is necessarily a simple open geodesic. We choose a lift of this simple geodesic $\tilde{\beta}$ and a lift $\tilde{\gamma}$ of $\gamma$ and observe that the orthogeodesic from $\tilde{\gamma}$ to $\tilde{\beta}$ has finite length. This orthogeodesic extends to an orthoray that enters the half-plane bounded by $\tilde{\beta}$. Pushing this orthoray to the quotient $X$ and noting that this orthoray exits the end $e$ proves item (2). That there is an intervals worth of such orthorays that hit $\tilde{\beta}$ follows from the general fact that the basepoint of an orthogeodesic between any two geodesics $\tilde{\gamma}$ to $\tilde{\beta}$ in $\mathbb{H}$ has an interval of orthorays that all intersect $\tilde{\beta}$ (See figure 2 ). Thus $2 \Longrightarrow 3$. The final implication $3 \Longrightarrow 1$ is clear.

We are now ready to prove Theorem 5.1.

Proof. For each $n$, let $\left\{Y_{n}\right\}_{n=1}^{\infty}$ be an exhaustion of $X^{\prime}$ by finite area geodesic subsurfaces which, to make the argument less cumbersome, we assume have the additional property that if a boundary geodesic of $Y_{n}$ is not a boundary geodesic of $X^{\prime}$ then it is not a boundary geodesic of $Y_{n+1}$. Now set $Z_{n+1}=Y_{n+1}-Y_{n}$ and note that in general $Z_{n}$ is a finite union of (possibly disconnected) geodesic subsurfaces. Fix $\gamma$ a simple closed oriented geodesic on $Y_{1}$ and choose an ordered countable dense subset $A=\left\{a_{n}\right\}_{n=1}^{\infty}$ of $\gamma$. If $\gamma$ is a boundary geodesic of $Y_{1}$, by possibly changing the orientation of $\gamma$, we may assume that $Y_{1}$ lies to the left of $\gamma$. Consider the orthoray $g_{1}$ that lies to the left of $\gamma$ and is based at $a_{1}$. If the ray stays inside $Y_{1}$ for all time or if it hits $\partial Y_{1}$ in a component that is a boundary geodesic 


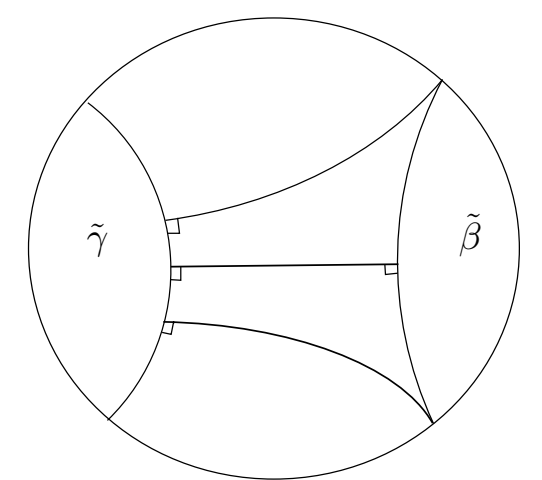

Figure 2. Interval of orthorays.

of $X^{\prime}$ then we do nothing and glue $Z_{2}$ any way we like. Otherwise, $g_{1}$ hits a component, say $\alpha$, of $\partial Y_{1}$, at a point we denote by $p_{1}$. Denote the angle $g_{1}$ makes with $\alpha$ by $\theta_{1}$. Let $Z_{2}^{*}$ be the component of $Z_{2}$ which contains the geodesic that will be identified with $\alpha$. From Lemma 5.2 the vector field $V_{\pi-\theta_{1}}$ based in $\alpha$ contains a Cantor set of vectors, whose associated geodesic ray stays in $Z_{2}$. Pick one and call it $v_{1}$. Now glue $Z_{2}$ to $Y_{1}$ along $\alpha$ so that the orthoray $g_{1}$ extends smoothly through $p_{1}$ and into $Z_{2}$ (see figure 3 ). By construction the ray $g_{1}$ stays inside $Y_{2}=Y_{1} \cup Z_{2}$ for infinite time. Next consider the orthoray $g_{2}$ with basepoint $a_{2}$ in the subsurface $Y_{2}$. As before if $g_{2}$ stays inside $Y_{2}$ or hits the $\partial Y_{2}$ in a boundary component of $X^{\prime}$ then we do nothing and just glue $Z_{3}$ anyway we like. Otherwise, as before we use Lemma 5.2 to glue $Z_{3}$ to $Y_{2}$ so that the orthoray $g_{2}$ extends smoothly into $Z_{3}$ and stays inside $Y_{3}=Y_{2} \cup Z_{3}$ for infinite time. We can continue this process ad infinitum so that we have constructed $X$ with specified twist parameters so that the orthoray which begins at $a_{n}$ stays in $Y_{n+1}$. Now using Lemma 5.3 we may conclude that all the infinite type ends of $X$ are not visible. Finally we add funnels to all the closed boundary geodesics of $X^{\prime}$ and conclude that the resulting surface is geodesically complete.
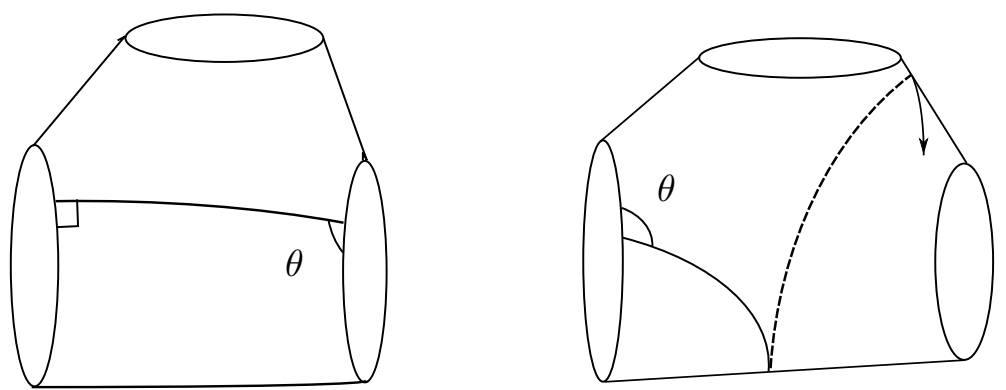

Figure 3. Angles match.

A flute surface is a sequence of pairs of pants glued in succession along common length boundaries. The flute surface as it stands is not necessarily geodesically complete but 
always has a natural geodesic completion by Theorem 3.4. A flute surface has genus zero, no non-planar ends, and space of ends homeomorphic to $\{1 / n\}_{n=1}^{\infty} \cup 0$. Denote the successive cuffs of the flute surface by $\left\{\alpha_{n}\right\}$ as in figure 4 . We say that a flute surface is tight if each of the pants holes that have not been glued along are in fact cusps.

Remark 5.4. A tight flute surface is geodesically complete if and only if its unique infinite type end is not visible.

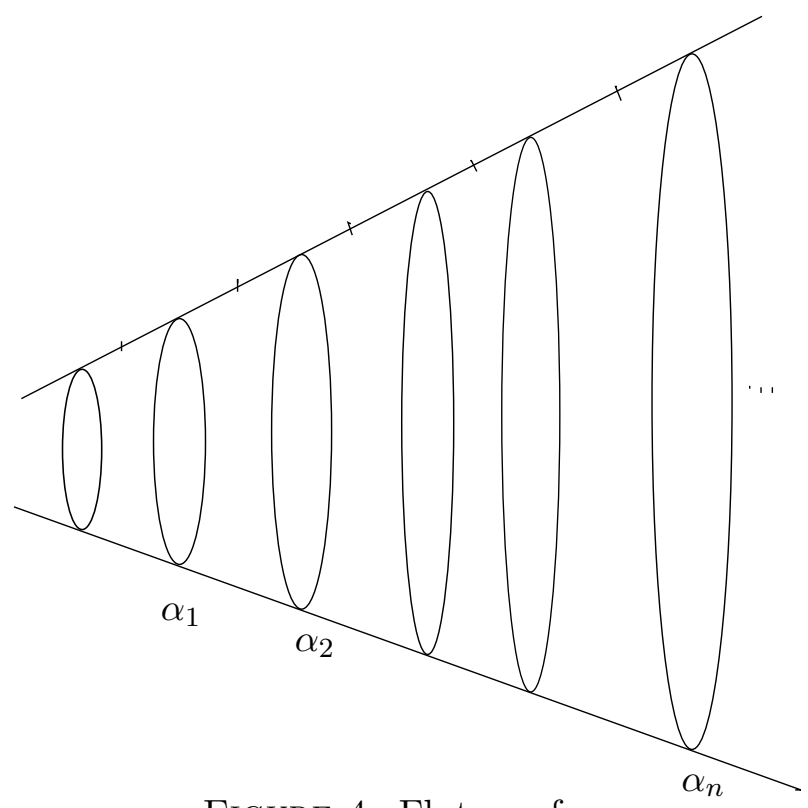

FiguRE 4. Flute surface.

Theorem 5.5. Fix any positive numbers $\left\{\ell_{n}\right\}$, where $\ell_{n} \rightarrow \infty$. There exists a tight flute surface of the first kind (that is geodesically complete) with $\ell\left(\alpha_{n}\right)=\ell_{n}$, for all $n$.

Proof. Recall that a flute surface has countably many isolated ends which converge to an infinite type end. We construct this surface $X^{\prime}$ out of tight pairs of pants, $\left\{P_{n}\right\}_{n=0}^{\infty}$, glued in succession. $P_{0}$ has cuff lengths, $\left(0,0, \ell_{1}\right)$ and then generally $P_{n}$ has cuff lengths $\left(\ell_{n}, 0, \ell_{n+1}\right)$ for $n=1,2,3, \ldots$. The gluing parameters are chosen as in Theorem 5.1. With these choices the infinite type end of $X^{\prime}$ is of the first kind, and hence all ends of $X$ are of the first kind. By Proposition 3.7, $X^{\prime}=X$ is a tight flute of the first kind.

Corollary 5.6. Let $X$ be the infinite genus surface with one end. There exists a geodesically complete hyperbolic structure on $X$ for which

(1) the hyperbolic structure is of the first kind. In particular, the infinite type end is not visible (complete),

(2) its length spectrum is discrete. That is, there are finitely many closed geodesics with length less than any given number. In particular, any pants decomposition is not upper bounded but is lower bounded. 
Proof. In the paper [8] it is shown that for any topological surface there exist hyperbolic structures with a discrete length spectrum. Moreover, the constructions are independent of the twist parameters. In particular, we can construct an infinite genus surface with one end using pairs of pants so that it admits a hyperbolic structure with a discrete length spectrum for any choice of twist parameters. Choosing the twist parameters as in Theorem 5.1 we are guaranteed that the infinite type end is not visible, and hence (since there are no other ends) the hyperbolic structure is of the first kind.

\section{Flute SURFACES With RAPID INCREASE IN CUFF LENGTHS}

We consider a tight flute surface (see figure 4). That is, we have a sequence $\left\{P_{n}\right\}$ of (tight) geodesic pairs of pants whose one cuff is a cusp and two other cuffs are geodesics $\alpha_{n}$ and $\alpha_{n+1}$. Except for the first pants which has two cusps. Denote by $l_{n}$ the length of $\alpha_{n}$. We choose one cuff of $P_{n}$ to be a cusp for the simplicity of the topology of the surface.

We glue $P_{n}$ to $P_{n+1}$ by identifying $\alpha_{n+1}$ on $P_{n}$ with $\alpha_{n+1}$ on $P_{n+1}$. The identification is given by a twist parameter $t_{n}$ with $0 \leq t_{n}<l_{n}$. We fix a choice of $t_{n}$ such that the surface obtained after all the identifications is complete (that is, no visible ends) which is possible by Theorem 2. Thus the obtained surface is a geodesically complete tight flute surface. There are many choices in the gluings such that the obtained surface is geodesically complete. We fix one such choice and denote the geodesically complete tight flute surface by $X_{0}$.

We next choose the cuff lengths $\left\{l_{n}\right\}$ of the pants $\left\{P_{n}\right\}$ to be rapidly increasing. That is, $\left\{l_{n}\right\}$ (strictly) monotonically goes to $\infty$ and

$$
\sum_{i=1}^{n} l_{i}=o\left(l_{n+1}\right) .
$$

\section{TEICHMÜLLER SPACES}

The quasiconformal Teichmüller space $T_{q c}\left(X_{0}\right)$ of a geodesically complete hyperbolic surface $X_{0}$ (without visible ends) consists of all quasiconformal mappings $f: X_{0} \rightarrow X$ modulo post compositions by conformal maps and homotopy. The Teichmüller distance between two points $\left[f: X_{0} \rightarrow X_{1}\right]$ and $\left[g: X_{0} \rightarrow X_{2}\right]$ is given by

$$
d_{T}([f],[g])=\frac{1}{2} \inf _{h \sim f \circ g^{-1}} \log K(h)
$$

where the infimum is over all quasiconformal maps $h$ homotopic to $f \circ g^{-1}$ and $K(h)$ is the quasiconformal constant of $h$.

The length spectrum Teichmüller space $T_{l s}\left(X_{0}\right)$ of a geodesically complete hyperbolic surface $X_{0}$ consists of all homeomorphisms $f: X_{0} \rightarrow X$ up to isometry and homotopy, where $X$ is a hyperbolic (not necessarily complete) surface with $\sup _{\alpha}\left|\log \frac{l_{X}(\alpha)}{l_{X_{0}}(\alpha)}\right|<\infty$ and the supremum is over all homotopy classes of simple closed curves $\alpha$. The length spectrum 
distance on $T_{l s}\left(X_{0}\right)$ is defined by

$$
d_{l s}([f],[g])=\frac{1}{2} \sup _{\alpha}\left|\log \frac{l_{f\left(X_{0}\right)}(\alpha)}{l_{g\left(X_{0}\right)}(\alpha)}\right|
$$

where the supremum is over all simple closed geodesics $\alpha$ on $X_{0}$.

The length spectrum Teichmüller space $T_{l s}\left(X_{0}\right)$ is complete in the length spectrum metric (cf. [4]) and $T_{q c}\left(X_{0}\right) \subset T_{l s}\left(X_{0}\right)$. When $X_{0}$ has a geodesic pants decomposition whose cuff lengths are bounded from above and from below then the length spectrum metric induces the same topology as the Teichmüller metric on $T_{q c}\left(X_{0}\right)$ (cf. [18]). When $X_{0}$ has a geodesic pants decomposition with upper bounded cuff lengths and a sequence of cuff lengths goes to zero, then the length spectrum metric is incomplete on $T_{q c}\left(X_{0}\right)$ (cf. [2]) and thus it does not induce the same topology as the Teichmüller metric. In the case of upper bounded cuff lengths, Teichmüller spaces $T_{q c}\left(X_{0}\right)$ and $T_{l s}\left(X_{0}\right)$ are parametrized by the Fenchel-Nielsen coordinates (cf. [3], [2]), the closure of $T_{q c}\left(X_{0}\right)$ inside $T_{l s}\left(X_{0}\right)$ is described in terms of the Fenchel-Nielsen coordinates (cf. [17]) and local biLipschitz structures of $T_{q c}\left(X_{0}\right)$ and $T_{l s}\left(X_{0}\right)$ is described using the Fenchel-Nielsen coordinates (cf. [3] and [17]). When $X_{0}$ has no geodesic pants decomposition with an upper bounded cuff lengths, the parametrization of $T_{q c}\left(X_{0}\right)$ using the Fenchel-Nielsen coordinates is rather challenging. In what follows we describe some aspects of the Fenchel-Nielsen coordinates when $X_{0}$ is a flute surface with rapidly increasing cuff lengths.

\section{The Closure of $T_{q c}\left(X_{0}\right)$}

In this section we assume that $X_{0}$ is the geodesically complete hyperbolic flute surface defined in section 6 . Namely, $X_{0}$ is obtained by gluing tight pairs of pants with cuff lengths $\left\{l_{n}\right\}$ satisfying

$$
\sum_{i=1}^{n} l_{i}=o\left(l_{n+1}\right) .
$$

In what follows we need the following lemma.

Lemma 8.1. Let $\Sigma$ be a geodesic pentagon with right angles at $A, B, C$ and $D$, and an ideal vertex at $E$. Let $a, b$ and $c$ be the lengths of the sides $A B, B C$ and $C D$, respectively. Let $d$ be the length of the geodesic segment orthogonal to both $A B$ and $D E$. Then, for $a>1$ large enough and $c>a$, there exist constants $C_{1}$ and $C_{2}$ such that

$$
C_{1}+c-a \leq d \leq C_{2}+c-a .
$$

Proof. Note that the segment orthogonal to $A B$ and $D E$ is necessarily inside the pentagon $A B C D E$. Denote by $A^{\prime}$ the endpoint in $A B$ and by $D^{\prime}$ the endpoint in $D E$ of the segment (cf. Figure 5). From the pentagon $\Sigma$, we get (cf. Beardon [9, page 159])

$$
\cosh a \cosh c+1=\sinh a \cosh b \sinh c
$$

which gives

$$
\cosh b=\frac{\cosh a \cosh c+1}{\sinh a \sinh c} .
$$


Further

$$
\frac{\cosh a \cosh c+1}{\sinh a \sinh c}=\frac{\left(1+e^{-2 a}\right)\left(1+e^{-2 c}\right)+4 e^{-(a+c)}}{\left(1-e^{-2 a}\right)\left(1-e^{-2 c}\right)}
$$

and Taylor's expansion gives, for some constant $C>0$,

$$
1+C e^{-2 a} \geq \cosh b \geq 1+e^{-2 a} .
$$

Consequently, for a constant $C^{\prime}>0$ which depends on $C$,

$$
C^{\prime} e^{-a} \geq \sinh b=\sqrt{\cosh ^{2} b-1} \geq \sqrt{2} e^{-a} .
$$

From the right angled pentagon $A^{\prime} B C D D^{\prime}$, we get (cf. Beardon [9, page 159])

$$
C^{\prime \prime} e^{-a} e^{c} \geq \cosh d=\sinh b \sinh c \geq \frac{\sqrt{2}}{4} e^{-a} e^{c}
$$

when $c$ is large enough which implies

$$
C^{\prime \prime \prime} e^{c-a} \geq e^{d} \geq \cosh d \geq \frac{\sqrt{2}}{4} e^{c-a} .
$$

Taking logarithms in the above inequality, we get

$$
C_{2}+c-a \geq d \geq \log \frac{\sqrt{2}}{4}+c-a .
$$

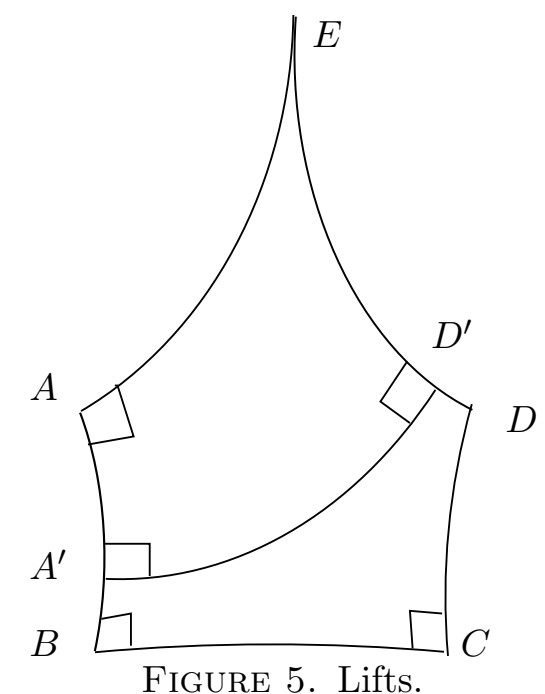

We study the closure $\overline{T_{q c}\left(X_{0}\right)}$ of the quasiconformal Teichmüller space $T_{q c}\left(X_{0}\right)$ for the length spectrum metric in the length spectrum Teichmüller space $T_{l s}\left(X_{0}\right)$. We establish 
Theorem 8.2. Let $X_{0}$ be a geodesically complete tight flute surface built by gluing pairs of pants with rapidly increasing cuff lengths $\left\{l_{n}\right\}$. Then the closure $\overline{T_{q c}\left(X_{0}\right)}$ of the quasiconformal Teichmüller space $T_{q c}\left(X_{0}\right)$ in the length spectrum metric contains all surfaces with the Fenchel-Nielsen coordinates $\left\{\left(l_{n}, t_{n}\right)\right\}_{n}$, where $-C l_{n} \leq t_{n} \leq C l_{n}$, for $C>0$, and the lengths $\left\{l_{n}\right\}$ correspond to a marked surface in $T_{q c}\left(X_{0}\right)$.

Proof. Denote by $\left\{P_{n}\right\}$ the family of tight geodesic pairs of pants that are used to obtain the flute surface $X_{0}$. Let $\alpha_{n}$ and $\alpha_{n+1}$ be the cuffs of $P_{n}$ that are not cusps. Then $\alpha_{n}$ has length $l_{n}$.

Let $\left\{\left(l_{n}, t_{n}^{\prime}\right)\right\}_{n \in \mathbb{N}}$ be the Fenchel-Nielsen coordinates of a marked surface $X$ in $T_{q c}\left(X_{0}\right)$. Define $t_{n}^{\prime \prime}=t_{n}-t_{n}^{\prime}$ for some $t_{n}$ with $-C l_{n} \leq t_{n} \leq C l_{n}$ and $C>0$. Let $f_{k}: X \rightarrow X_{k}$ be a quasiconformal marking map from $X$ to the surface $X_{k}$ obtained by twists $t_{i}^{\prime \prime}$ around $\alpha_{i}$ on the surface $X$ for $i=1,2, \ldots, k$. It is clear that $f_{k}$ can be chosen to be a quasiconformal map since we twist around only finitely many geodesics (cf. [16]). We prove that the marked surfaces $X_{k}$ converge in the length spectrum metric to the surface $X^{*}$ whose Fenchel-Nielsen coordinates are $\left\{\left(l_{n}, t_{n}\right)\right\}_{n \in \mathbb{N}}$.

We divide each pair of pants $P_{n}$ into two pentagons with one ideal vertex by three simple geodesic arcs: the first arc, denoted by $\beta_{n}$, is orthogonal to $\alpha_{n}$ and $\alpha_{n+1}$ at its endpoints, the second arc, denoted by $\beta_{n}^{1}$, is orthogonal to $\alpha_{n}$ at its endpoint and it finishes in the cusp, and the third arc, denoted by $\beta_{n}^{2}$, is orthogonal to $\alpha_{n+1}$ at its endpoint and it also finishes in the cusp. Note that the two pentagons are isometric and that they have four straight angles and one zero angle, i.e. one ideal vertex. Let $b_{n}$ be the length of $\beta_{n}$.

Let $\gamma$ be an arbitrary simple closed geodesic on $X$. If $\gamma$ does not intersect any $\left\{\alpha_{i}\right\}$ for $i \geq k$ then $l_{X_{k}}(\gamma)=l_{X^{*}}(\gamma)$ and there is nothing to be proved in this case.

Assume that $\gamma$ intersects $\alpha_{i}$ for $i=i_{0}, i_{0}+1, \ldots, j_{0}$ with $k<j_{0}$. In this case we need to estimate the size of $l_{X^{*}}(\gamma)$ compared $l_{X_{k}}(\gamma)$. We first estimate the size of $l_{X_{k}}(\gamma)$. Note that $\gamma$ intersects the pants $P_{j_{0}}$ whose boundary geodesics are $\alpha_{j_{0}}$ and $\alpha_{j_{0}+1}$. By assumption, $\gamma$ does not intersect $\alpha_{j_{0}+1}$ which implies that $\gamma$ enters and exists the pants $P_{j_{0}}$ through $\alpha_{j_{0}}$. This implies that $\gamma$ necessarily intersects the geodesic arc $\beta_{j_{0}}^{2}$ orthogonal to $\alpha_{j_{0}+1}$ that ends in the puncture because otherwise $\gamma$ could be homotoped such that it does not intersect $\alpha_{j_{0}}$ which is impossible.

We estimate the length of $\gamma \cap P_{j_{0}}$. Consider the lift of the situation to the universal covering $\mathbb{H}$. Fix a single component $\tilde{\alpha}_{j_{0}}$ of the lift of $\alpha_{j_{0}}$ to $\mathbb{H}$. Denote by $\Sigma_{1}$ and $\Sigma_{2}$ the two pentagons that the pants $P_{j_{0}}$ is divided into. Consider all lifts of $\Sigma_{1}$ and $\Sigma_{2}$ that have one side on $\tilde{\alpha}_{j_{0}}$. A lift $\tilde{\gamma}$ of $\gamma$ connects $\tilde{\alpha}_{j_{0}}$ with a lift $\tilde{\beta}_{j_{0}}^{2}$ of $\beta_{j_{0}}^{2}$ that belongs to a lift of $\Sigma_{1}$ or $\Sigma_{2}$ with one boundary side on $\tilde{\alpha}_{j_{0}}$. The length of the segment of $\tilde{\gamma}$ that has one endpoint on $\tilde{\alpha}_{j_{0}}$ and the other endpoint on $\tilde{\beta}_{j_{0}}^{2}$ is greater than the length of the common perpendicular geodesic arc to $\tilde{\alpha}_{j_{0}}$ and $\tilde{\beta}_{j_{0}}^{2}$. It is immediate that the common perpendicular geodesic arc $p$ lies in a single lift of one of the two pentagons (cf. Figure 2). Then Lemma 8.1 implies that the length of the common perpendicular arc $p$ is at least $C_{1}+\frac{l_{j_{0}+1}-l_{j_{0}}}{2}$. It follows that

$$
l_{X^{*}}(\gamma), l_{X_{k}}(\gamma) \geq C_{1}+l_{j_{0}+1}-l_{j_{0}}
$$




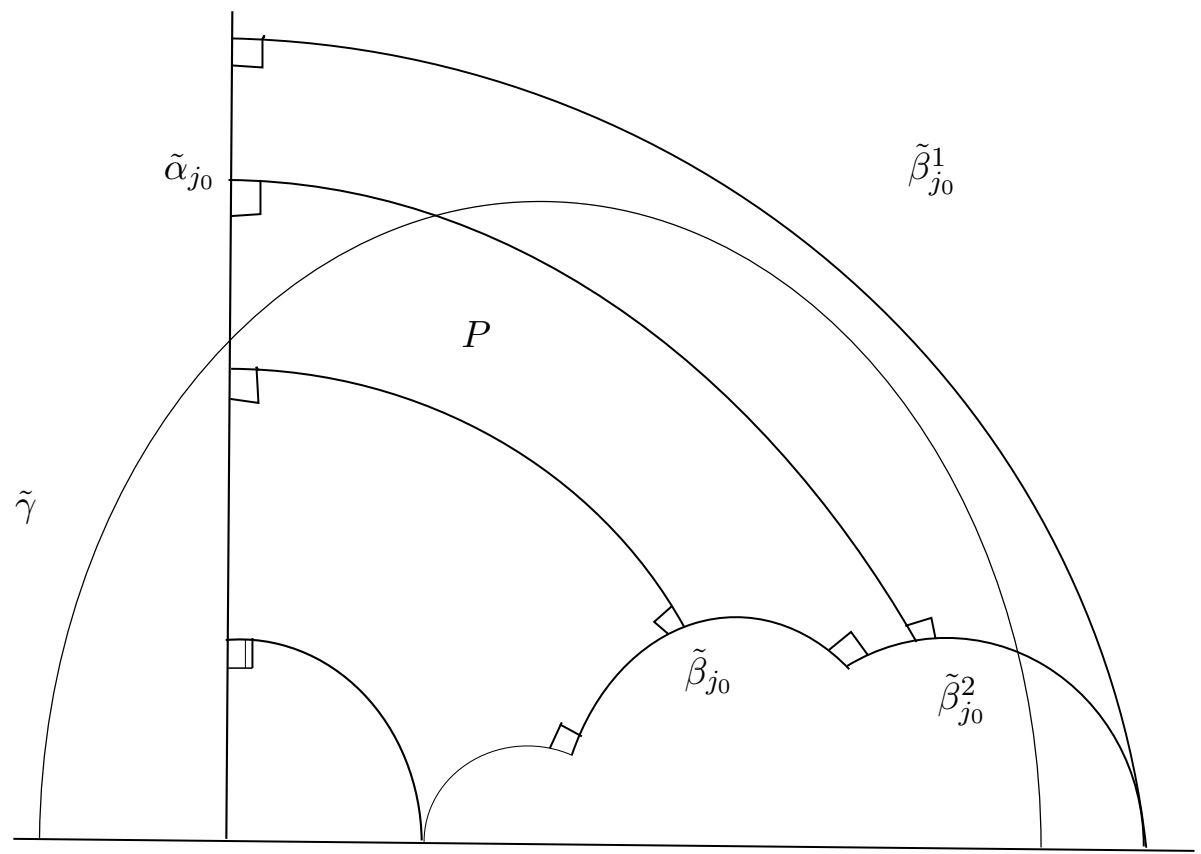

FiguRE 6. Lifts.

Since $X^{*}$ is obtained by twisting around $\alpha_{n}$ by the amount $t_{n}^{\prime \prime}$ and $\left|t_{n}^{\prime \prime}\right| \leq(C+1) l_{n}$, we obtain

$$
l_{X^{*}}(\gamma) \leq l_{X_{k}}(\gamma)+\sum_{i=i_{0}}^{j_{0}}\left|t_{i}^{\prime \prime}\right| \leq l_{X_{k}}(\gamma)+o\left(l_{j_{0}+1}\right)
$$

We get

$$
\frac{l_{X^{*}}(\gamma)}{l_{X_{k}}(\gamma)} \leq 1+\frac{1}{l_{X_{k}}(\gamma)} o\left(l_{j_{0}+1}\right) \leq 1+\frac{o\left(l_{j_{0}+1}\right)}{C_{1}+l_{j_{0}+1}-l_{j_{0}}} \leq 1+\frac{o\left(l_{k+1}\right)}{C_{1}+l_{k+1}-l_{k}} \rightarrow 1
$$

as $k \rightarrow \infty$ uniformly in $\gamma$.

Notice that $X_{k}$ is obtained by twisting $-t_{i}^{\prime \prime}$ along $\alpha_{i}$ for $i>k$. If $\gamma$ is a simple closed geodesic that intersects $\alpha_{i}$ for $i=i_{0}, i_{0}+1, \ldots, j_{0}$. If $j_{0} \leq k$ then $l_{X_{k}}(\gamma) / l_{X^{*}}(\gamma)=1$. If $j_{0}>k$ then

$$
l_{X_{k}}(\gamma) \leq l_{X^{*}}(\gamma)+\sum_{i=i_{0}}^{j_{0}}\left|t_{i}^{\prime \prime}\right| \leq l_{X^{*}}(\gamma)+o\left(l_{j_{0}+1}\right)
$$

which implies as before that

$$
\frac{l_{X_{k}}(\gamma)}{l_{X^{*}}(\gamma)} \leq 1+\frac{1}{l_{X^{*}}(\gamma)} o\left(l_{j_{0}+1}\right) \leq 1+\frac{o\left(l_{j_{0}+1}\right)}{C_{1}+l_{j_{0}+1}-l_{j_{0}}} \leq 1+\frac{o\left(l_{k+1}\right)}{C_{1}+l_{k+1}-l_{k}} \rightarrow 1
$$

as $k \rightarrow \infty$ uniformly in $\gamma$.

We obtained the convergence of $X_{k} \in T_{q c}\left(X_{0}\right)$ to $X^{*}$ in the length spectrum metric. 
We need the following two lemmas.

Lemma 8.3. Let $\Sigma$ be a pentagon with four right angles and one ideal vertex, i.e. zero angle. Let $a, b$ and $c$ be the lengths of three finite sides of $\Sigma$ in the counterclockwise order. Assume that $c>a>1, a \rightarrow \infty$ and $c / a \rightarrow \infty$. Then

$$
b=2 e^{-a}+o\left(e^{-a}\right)
$$

where $o\left(e^{-a}\right) / e^{-a} \rightarrow 0$ as $a \rightarrow \infty$.

Proof. We have (cf. [9])

$$
\cosh a \cosh c+1=\sinh a \cosh b \sinh c
$$

which gives

$$
\cosh b=\frac{\cosh a \cosh c+1}{\sinh a \sinh c}=\frac{\left(1+e^{-2 a}\right)\left(1+e^{-2 c}\right)+4 e^{-(a+c)}}{\left(1-e^{-2 a}\right)\left(1-e^{-2 c}\right)}=1+2 e^{-2 a}+o\left(e^{-2 a}\right) .
$$

and the result follows.

Lemma 8.4. Let $\mathcal{Q}$ be a hyperbolic quadrilateral with three right angles and a fourth angle $0<\phi<\frac{\pi}{2}$. Let $a_{1}$ and $a_{2}$ be the lengths of the sides of $\mathcal{Q}$ with two right angles, and let $b_{1}$ and $b_{2}$ be the lengths of the opposite sides, respectively. Then

$$
\sinh b_{1}=\sinh a_{1} \cosh b_{2}
$$

and

$$
\tanh a_{1} \sinh b_{2} \tan \phi=1 .
$$

Proof. From [9, page 157, Theorem 7.17.1], we have

$$
\begin{aligned}
& \sinh a_{1} \sinh a_{2}=\cos \phi \\
& \cosh a_{1}=\cosh b_{1} \sin \phi \\
& \cosh a_{2}=\cosh b_{2} \sin \phi
\end{aligned}
$$

Using (6) and $\sin ^{2} \phi+\cos ^{2} \phi=1$, we get

which implies

$$
\sinh ^{2} a_{1} \sinh ^{2} a_{2}+\frac{\cosh ^{2} a_{1}}{\cosh ^{2} b_{1}}=1
$$

$$
\sinh ^{2} a_{1} \sinh ^{2} a_{2} \cosh ^{2} b_{1}+\cosh ^{2} a_{1}=\cosh ^{2} b_{1} .
$$

Substituting $\sinh ^{2} a_{2}=\cosh ^{2} a_{2}-1$ above we get

$$
\sinh ^{2} a_{1} \cosh ^{2} a_{2} \cosh ^{2} b_{1}-\sinh ^{2} a_{1} \cosh ^{2} b_{1}+\cosh ^{2} a_{1}=\cosh ^{2} b_{1} .
$$

By diving the third equation with the second equation in $(6)$, we get $\cosh a_{2}=\frac{\cosh b_{2}}{\cosh b_{1}} \cosh a_{1}$ and substituting above gives

$$
\sinh ^{2} a_{1}\left(\frac{\cosh b_{2}}{\cosh b_{1}} \cosh a_{1}\right)^{2} \cosh ^{2} b_{1}-\sinh ^{2} a_{1} \cosh ^{2} b_{1}+\cosh ^{2} a_{1}=\cosh ^{2} b_{1}
$$

which in turn gives

$$
\sinh ^{2} a_{1} \cosh ^{2} b_{2} \cosh ^{2} a_{1}-\sinh ^{2} a_{1} \cosh ^{2} b_{1}-\cosh ^{2} b_{1}+\cosh ^{2} a_{1}=0 .
$$


Since $\sinh ^{2} a_{1}+1=\cosh ^{2} a_{1}$, the above gives

$$
\sinh ^{2} a_{1} \cosh ^{2} b_{2} \cosh ^{2} a_{1}-\cosh ^{2} a_{1} \cosh ^{2} b_{1}+\cosh ^{2} a_{1}=0 .
$$

Finally, dividing with $\cosh ^{2} a_{1}$ gives

$$
\sinh ^{2} a_{1} \cosh ^{2} b_{2}-\cosh ^{2} b_{1}+1=0
$$

and the first formula follows easily.

To prove the second formula, note that (6) implies

$$
\sinh ^{2} a_{2}=\frac{\cos ^{2} \phi}{\sinh ^{2} a_{1}}
$$

and then

$$
\cosh ^{2} a_{2}=\sinh ^{2} a_{2}+1=\frac{\cos ^{2} \phi}{\sinh ^{2} a_{1}}+1 .
$$

By using (6) above, we get

$$
\frac{\cos ^{2} \phi}{\sinh ^{2} a_{1}}+1=\cosh ^{2} b_{2} \sin ^{2} \phi
$$

which gives

$$
\cos ^{2} \phi+\sinh ^{2} a_{1}=\sinh ^{2} a_{1} \cosh ^{2} b_{2} \sin ^{2} \phi .
$$

Further

$$
\cos ^{2} \phi+\sinh ^{2} a_{1} \cos ^{2} \phi=\sinh ^{2} a_{1} \cosh ^{2} b_{2} \sin ^{2} \phi-\sinh ^{2} a_{1} \sin ^{2} \phi
$$

and then

$$
\cosh ^{2} a_{1} \cos ^{2} \phi=\sinh ^{2} a_{1} \sinh ^{2} b_{2} \sin ^{2} \phi
$$

and the second formula follows.

The following theorem proves that pointwise convergence of twists implies the convergence in the length spectrum metric when the lengths of the cuffs in the pants decomposition are fixed.

Theorem 8.5. Let $X_{0}$ be a geodesically complete tight flute surface with twists $\left\{t_{n}\right\}$ and rapidly increasing cuff lengths $\left\{l_{n}\right\}$. Let $X_{k}$ be marked hyperbolic surface with cuff lengths equal to $\left\{l_{n}\right\}$ and twists $t_{X_{k}}\left(\alpha_{n}\right)=t_{n}+O\left(l_{n}\right)$. If $\lim _{k \rightarrow \infty} t_{X_{k}}\left(\alpha_{n}\right)=t_{n}$ for each $n$, then $X_{k}$ converges to $X_{0}$ in the length spectrum metric.

Proof. Let $\epsilon>0$ be given. We need to prove that $\left|\frac{l_{X_{k}}(\beta)}{l_{X_{0}}(\beta)}-1\right|<\epsilon$ for all simple closed geodesics $\beta$ on $X_{0}$ and for all $k \geq k_{0}(\epsilon)$, where $k_{0}(\epsilon)>0$ depends on $\epsilon$.

Indeed, let $\beta$ be a simple closed geodesic on $X_{0}$. If $\beta$ is a cuff of the pants decomposition, then $l_{X_{0}}(\beta)=l_{X_{k}}(\beta)$ and $\left|\frac{l_{X_{k}}(\beta)}{l_{X_{0}}(\beta)}-1\right|=0$ for all $k$.

If $\beta$ transversely intersects cuffs, let $\alpha_{n_{0}}$ be the cuff with the largest index that $\beta$ intersects. Then we have

$$
l_{X_{0}}(\beta)-\sum_{j=1}^{n_{0}}\left|t_{X_{k}}\left(\alpha_{j}\right)-t_{j}\right| \leq l_{X_{k}}(\beta) \leq l_{X_{0}}(\beta)+\sum_{j=1}^{n_{0}}\left|t_{X_{k}}\left(\alpha_{j}\right)-t_{j}\right|
$$


and dividing it with $l_{X_{0}}(\beta)$ we get

$$
1-\frac{\sum_{j=1}^{n_{0}}\left|t_{X_{k}}\left(\alpha_{j}\right)-t_{j}\right|}{l_{X_{0}}(\beta)} \leq \frac{l_{X_{k}}(\beta)}{l_{X_{0}}(\beta)} \leq 1+\frac{\sum_{j=1}^{n_{0}}\left|t_{X_{k}}\left(\alpha_{j}\right)-t_{j}\right|}{l_{X_{0}}(\beta)} .
$$

As in the proof of Theorem 8.2 , we have

$$
l_{X_{0}}(\beta) \geq l_{n_{0}+1}-l_{n_{0}}+C .
$$

Note that

$$
\sum_{j=1}^{n_{1}}\left|t_{X_{k}}\left(\alpha_{j}\right)-t_{j}\right|=o\left(l_{n_{1}+1}\right)
$$

and we can choose $n_{1}=n_{1}(\epsilon)$ such that

$$
1-\epsilon<\frac{\sum_{j=1}^{n}\left|t_{X_{k}}\left(\alpha_{j}\right)-t_{j}\right|}{l_{n+1}-l_{n}+C}<1+\epsilon
$$

for all $n \geq n_{1}$.

Since $t_{X_{k}}\left(\alpha_{n}\right) \rightarrow t_{n}$ as $k \rightarrow \infty$ for each $n$, it follows that for any $n_{0} \leq n_{1}$ the sum $\sum_{j=1}^{n_{0}}\left|t_{X_{k}}\left(\alpha_{j}\right)-t_{j}\right|<\epsilon$ for all $k \geq k_{0}=k_{0}(\epsilon)$. Therefore, $\frac{l_{X_{k}}(\beta)}{l_{X_{0}}(\beta)}$ is $\epsilon$-close to 1 for $n_{0} \leq n_{1}$ with $k \geq k_{0}$ large enough.

If $n_{0}>n_{1}$ then

$$
\frac{\sum_{j=1}^{n_{0}}\left|t_{X_{k}}\left(\alpha_{j}\right)-t_{j}\right|}{l_{X_{0}}(\beta)} \leq \frac{o\left(l_{n_{0}+1}\right)}{l_{n_{0}+1}-l_{n_{0}}+C} \leq \frac{o\left(l_{n_{1}+1}\right)}{l_{n_{1}+1}-l_{n_{1}}+C}<\epsilon
$$

by the choice of $n_{1}$ for all $k$. It follows that $\left|\frac{l_{X_{k}}(\beta)}{l_{X_{0}}(\beta)}-1\right|<\epsilon$ when $k>k_{0}$. The convergence in the length spectrum metric is proved.

Using Theorem 8.2 we obtain

Theorem 8.6. If $X_{0}$ is a geodesically complete tight flute surface with rapidly increasing cuff lengths, then the length spectrum Teichmüller space $T_{l_{s}}\left(X_{0}\right)$ is strictly larger than the quasiconformal Teichmüller space $T_{q c}\left(X_{0}\right)$. More precisely, $\overline{T_{q c}\left(X_{0}\right)}-T_{q c}\left(X_{0}\right)$ is non-empty.

Proof. Let $\left\{t_{n}\right\}$ and $\left\{l_{n}\right\}$ be the twists and the length parameters of $X_{0}$ for the pants decomposition $\mathcal{P}=\left\{\alpha_{n}\right\}$ as above. Define $t_{k}\left(\alpha_{n}\right)=t_{n}+\left|l_{n}\right|$ if $k \leq n$, and define $t_{k}\left(\alpha_{n}\right)=t_{n}$ if $k>n$. Define $l_{k}\left(\alpha_{n}\right)=l_{n}$ for all $k, n$. The marked surface $X_{k}$ with the Fenchel-Nielsen coordinates $\left\{\left(t_{k}\left(\alpha_{n}\right), l_{k}\left(\alpha_{n}\right)\right)\right\}$ is a quasiconformal deformation of $X_{0}$. Indeed, the twists are positive and there are only finitely many of them which implies that the left earthquake given by the positive twists has Thurston bounded earthquake measure. Then the left earthquake induces a quasiconformal deformation (cf. [16]).

Let $X_{0}^{\prime}$ be the surface with lengths $\left\{l_{n}\right\}$ and twists $\left\{t_{n}+\left|l_{n}\right|\right\}$. Then $X_{k}$ converges to $X_{0}^{\prime}$ as $k \rightarrow \infty$ in the length spectrum metric because $t_{X_{k}}\left(\alpha_{n}\right)=t_{X_{0}^{\prime}}\left(\alpha_{n}\right)+O\left(l_{n}\right)$ for all $k, n$ and $t_{X_{k}}\left(\alpha_{n}\right)=t_{X_{0}^{\prime}}\left(\alpha_{n}\right)$ for $n \leq k$ (cf. Theorem 8.5). However, the limit $X_{0}^{\prime}$ is a not a quasiconformal deformation of $X_{0}$ since it is obtained by left earthquake with unbounded Thurston's norm (cf. [16]). 


\section{REFERENCES}

[1] Lars Ahlfors, Complex Analysis, McGraw Hill, 3rd ed., 1979.

[2] D. Alessandrini, L. Liu, A. Papadopoulos and W. Su, On the inclusion of the quasiconformal Teichmüller space into the length-spectrum Teichmüller space, preprint, arXiv:1201.6030.

[3] D. Alessandrini, L. Liu, A. Papadopoulos and W. Su, On various Teichmüller spaces of a surface of infinite topological type, Proc. Amer. Math. Soc. 140 (2012), 561-574.

[4] D. Alessandrini, L. Liu, A. Papadopoulos, W. Su and Z. Sun, On Fenchel-Nielsen coordinates on Teichmüller spaces of surfaces of infinite type, Ann. Acad. Sci. Fenn. Math. 36 (2011), no. 2, 621-659.

[5] V. Alvarez and J.M. Rodriguez, Structure theorems for Riemann and topological surfaces, J. London Math. Soc. (2) 69 (2004), 153-168.

[6] A. Basmajian, Hyperbolic structures for surfaces of infinite type, Trans. Amer. Math. Soc. 336, no. 1, March 1993, 421-444.

[7] A. Basmajian, Large parameter spaces of quasiconformally distinct hyperbolic structures, J. Anal. Math. 71 (1997), 75-85.

[8] A. Basmajian and Y. Kim, Geometrically infinite surfaces with discrete length spectra, Geom. Dedicata 137 (2008), 219-240.

[9] A. Beardon, The geometry of discrete groups, Graduate Texts in Mathematics, 91. Springer-Verlag, New York, 1983.

[10] P. Buser, Geometry and spectra of compact Riemann surfaces, Progress in Mathematics, 106. Birkhuser Boston, Inc., Boston, MA, 1992. xiv+454 pp. ISBN: 0-8176-3406-1

[11] D. B. A. Epstein, A. Marden ad V. Markovic, Quasiconformal homeomorphisms and the convex hull boundary, Ann. of Math. (2) 159 (2004), no. 1, 305-336.

[12] Andrew Haas and Perry Susskind, The geometry at infinity of a hyperbolic Reimann surface of infinite type, Geometriae Dedicata (2007), Volume 130, Issue 1, pp 1-24.

[13] E. Kinjo, On Teichmüller metric and the length spectrums of topologically infinite Riemann surfaces, Kodai Math. J. 34 (2011), no. 2, 179-190.

[14] K. Matsuzaki, A countable Teichmüller modular group. T. AMS., 357, 8 (2004), 3119-3131.

[15] I. Richards, On the classification of noncompact surfaces, Trans. Amer. Math. Soc. 106 (1963) 259-269.

[16] D. Šarić, Real and Complex Earthquakes, Trans. Amer. Math. Soc. 358 (2006), no. 1, 233-249.

[17] D. Šarić, Fenchel-Nielsen coordinates on upper bounded pants decompositions, to appear Math. Proc. Cambridge Philos. Soc., arXiv:1209.5819

[18] H. Shiga, On a distance defined by the length spectrum of Teichmüller space, Ann. Acad. Sci. Fenn. Math. 28 (2003), no. 2, 315326.

[19] S. Wolpert. The length spectra as moduli for compact Riemann surfaces, Ann. of Math. (2) 109 (1979), no. 2, 323-351.

Ara Basmajian, Department of Mathematics, Graduate Center and Hunter College, CUNY, abasmajian@gc.cuny.edu

Dragomir Šarić, Department of Mathematics, Graduate Center and Queens College, CUNY, Dragomir.Saric@qc.cuny.edu 